\title{
Successful implementation of remote video consultations for patients receiving home parenteral nutrition in a national UK Centre
}

\author{
Anabelle Cloutier (D) , ${ }^{1}$ Ashley Bond (D) , ${ }^{1}$ Michael Ian Taylor, ${ }^{1}$ \\ Joanne Ablett, ${ }^{1}$ Antje Teubner, ${ }^{1}$ Kirstine Farrer, ${ }^{1}$ Gavin Leahy, ${ }^{1}$ \\ Arun Abraham, ${ }^{1}$ Simon Lal ${ }^{1,2}$
}

${ }^{1}$ Intestinal Failure Unit, Salford Royal NHS Foundation Trust,

Salford, UK

2University of Manchester, Manchester, UK

\section{Correspondence to}

Dr Anabelle Cloutier, Intestinal Failure Unit, Salford Royal NHS Foundation Trust, Salford M6 8HD, UK; anabelle.cloutier@srft. nhs.uk

Received 9 May 2019 Revised 25 September 2019 Accepted 30 September 2019 Published Online First 14 October 2019

\section{Check for updates}

(c) Author(s) (or their employer(s)) 2020. No commercial re-use. See rights and permissions. Published by BMJ.

To cite: Cloutier $A$,

Bond A, Taylor MI, et al.

Frontline Gastroenterology

2020;11:280-284.

\section{ABSTRACT}

Rationale Our intestinal failure unit provides care for patients from a wide geographical area. Patients dependent on home parenteral nutrition (HPN) are routinely reviewed in the clinic at 3-6 monthly intervals. Between March 2008 and 2015, we noted a significant rise in the number of patients under our care, with an associated $51 \%$ increase in clinic appointments offered. We evaluated whether telemedicine would provide a strategy to reduce patients' need to travel while maintaining safe clinical standards.

Methods Implementation began in December 2015 via patient consultation and small tests of change. Clinical data were obtained from a prospectively maintained database. Remote video consultation discussions were carried out via internet video call service (Skype). An anonymous satisfaction questionnaire was offered to patients for completion following consultation. The number of miles saved by obviating the need to attend hospital was calculated for each patient.

Results During the study period, patients receiving HPN rose by $18 \%$ to 288 . Twenty-five patients used telemedicine for HPN followup, three of these for follow-up with the psychologist. By avoiding hospital attendance, this saved a mean travel distance of 56.7 miles with a total of 18346.6 cumulative miles saved. Sixty-three per cent of patients rated their satisfaction with the system at $\geq 90 \%$, with a mean satisfaction of $85 \%$. Eight percent of the telemedicine cohort was admitted with an HPN complication, compared with an admission rate of $24 \%$ for the whole HPN cohort. One emergency admission was avoided.

\section{Significance of this study}

What is already known on this topic

- Medicine has become highly specialised. However, care for specific diseases, such as intestinal failure, is offered in relatively few locations. Unfortunately, some patients cannot access specialised centres because of economical and geographical barriers. In order to offer equal access to high-quality care, many centres across the world have evaluated remote video consultation.

What this study adds

- Only a few papers have studied remote video consultation for the follow-up of patients dependant on parenteral nutrition. To our knowledge, this is the first report evaluating the remote psychological management of patients receiving home parenteral nutrition.

\section{How might it impact on clinical} practice in the foreseeable future?

- Remote video consultation might increase access to specialised care for patients with chronic illness. It may also improve patients' quality of life by avoiding long journeys to and from the hospital.

Conclusion Telemedicine can obviate the need for clinic attendance in HPN-dependent patients, so reducing the need of individuals with chronic illness to travel while maintaining standards for follow-up.

\section{INTRODUCTION}

Intestinal failure (IF) is defined as a 'reduction in the functioning gut mass 
below the minimal amount necessary for adequate digestion and absorption of nutrients'. ${ }^{1}$ It can be further divided into three types, depending on the chronicity of the disease; type I is self-limiting IF often following abdominal surgery; type II is a prolonged acute, metabolically unstable state, while the type III is a chronic disease requiring long-term home parenteral nutrition (HPN). ${ }^{2} 3$ Owing to the complexity of this disease, international guidance for the management of IF dictates that such patients are cared for in dedicated specialist centres, ${ }^{4}$ which are only available in a few locations. Hence, patients often live at a significant distance from these centres. All contemporary HPN guidelines further recommend the expertise of a multidisciplinary nutrition support team for these patients with regular contacts and monitoring every 3-6 months. ${ }^{45}$ Routine practice at the authors' centre reflected these guidelines, with patients attending hospital appointments for clinical, nutritional and blood assessments on a 3-6 monthly basis when stable.

Even though type III IF is a relatively rare disease with an estimated prevalence of 5-80 cases per million population, ${ }^{467}$ these numbers are growing in the UK and elsewhere ${ }^{68}$ with a $102 \%$ increase in adults dependent on HPN in the UK noted between 2011 and $2015 .^{8}$ Telemedicine provides a strategy for achieving accessibility of care while reducing travel needs and potentially maintaining or even improving the satisfaction of patients. ${ }^{9}$ The purpose of this study was to determine whether telemedicine can be used safely and effectively in a cohort of individuals dependent on HPN managed at a large national IF referral centre and to calculate the potential number of miles saved by avoiding travel to the hospital.

\section{METHODS}

The telemedicine remote consultation programme was launched in December 2015 in a national UK IF referral centre. Quality improvement (QI) methodology was used to implement the project. ${ }^{10}$ The video service Skype was selected as a communication tool, as it was deemed to be readily accessible to patients. Tests between the IF unit's staff (onsite and hospital to home), non-clinical trials with a patient at home and then clinical tests were made before implementation of the project. Patients deemed to be clinically stable (eg, no acute ongoing medical issues and no recent (within the last 6 months) changes in the parenteral nutrition script) were offered the option of participating in the telemedicine consultation programme at a routine face-to-face clinic appointment. Patients were offered this service if they had access and the ability to use web-based applications. Patients used their own electronic device in their home environment to interact with one of the doctors specialising in HPN, replacing the need to attend a follow-up appointment. The telemedicine system was also used by the IF unit's psychologist for patients who were unable to come to clinic to complete their therapy following hospitalisation, mainly due to the distance between home and hospital. Figure 1 details the process involved in telemedicine service development.

The interview was conducted in a private manner (patient at home, clinician in an enclosed office without interruption). Following consultation, an outcome letter was dictated to the general practitioner (GP). The patient's blood tests were checked by their GP after the telemedicine consultation and forwarded to the IF team. Patients were asked to attend the IF outpatient clinic at least once a year. Patients were

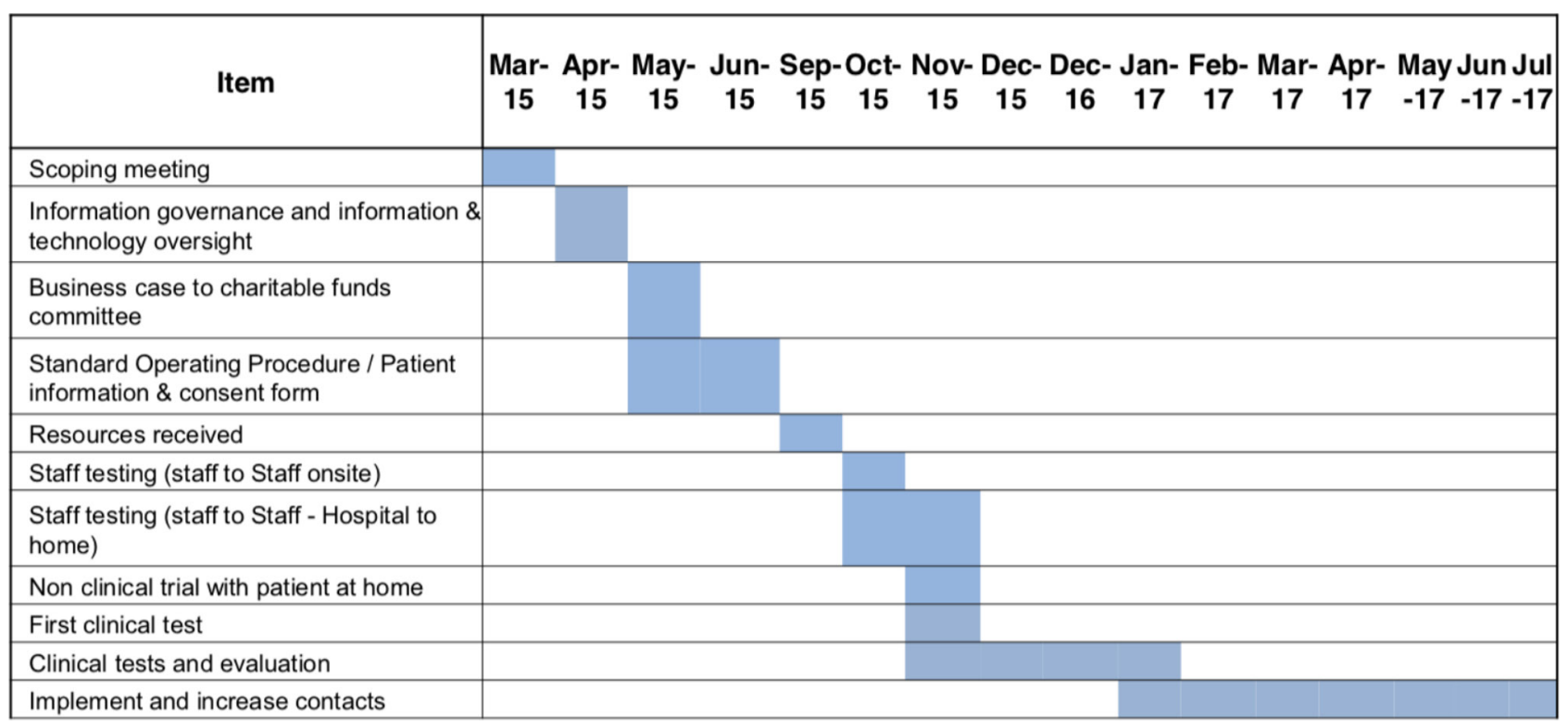

Figure 1 Implement and evaluate a remote consultation for patients dependent on home parenteral nutrition. 


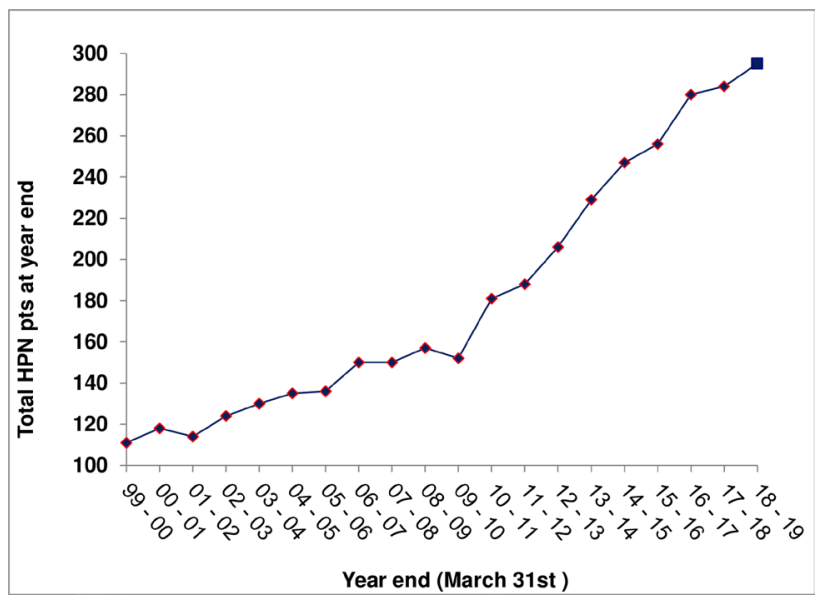

Figure 2 Number of HPN-dependent patients. HPN, home parenteral nutrition.

subsequently asked to rate their experience on a 1-10 visual analogue scale and also comment on their experience in an anonymous manner. Doctors rated the safety and reliability of consultation on a Likert scale. Clinical data such as the distance travelled by patients and any complications related to HPN during the study period were obtained from a prospectively maintained database as part of European Society for Clinical Nutrition and Metabolism(ESPEN) audit standards.

\section{RESULTS}

During the study period, December 2015-December 2018, there was a steady increase in the number of HPN-dependent patients managed at the IF centre (figure 2). Twenty-five patients used the telemedicine service during this period, totalling 105 contacts. Twenty-two patients used Skype for the HPN clinical follow-up (74 contacts), while three patients used it for psychological follow-up and intervention (31 contacts). One patient, with an acute clinical issue, was accompanied by their GP at home during the consultation. At first appointment, patients were aged from 25 to 76 years old (median age 60, 17 female).

The mean distance travelled (one way) to a hospital clinic appointment by the entire cohort of HPNdependent patients $(n=288)$ managed at our centre

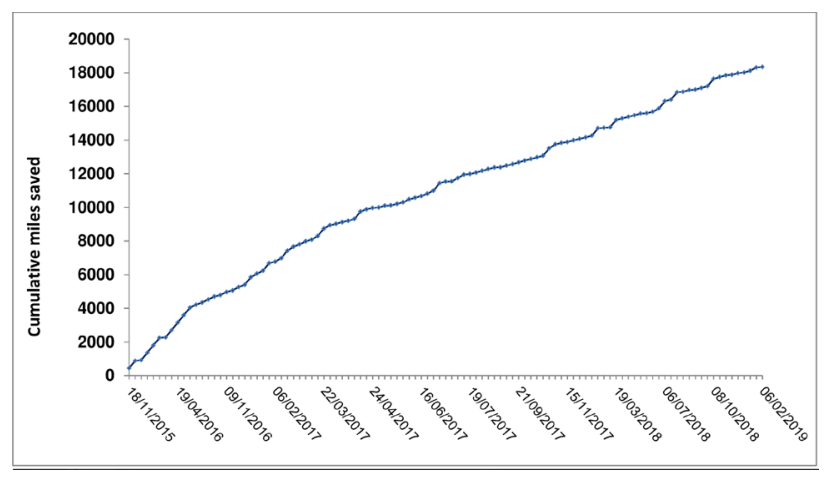

Figure 3 Accumulative (miles) travel saved. was 40.8 miles (range: $1.8-313.8$ ). The mean potential distance that those using the telemedicine service (one way) to attend a clinic appointment was 56.7 miles (range: 5.0-220.9); thus, cumulative miles saved by the telemedicine service by avoiding outpatient attendance were 18346.6 (figure 3). Doctors rated more than $94 \%$ of the consultations successful. Sixtythree percent of patients rated their satisfaction with the system at $\geq 90 \%$ on a visual analogue scale, with a mean satisfaction of $85 \%$. As an example, a patient mentioned the benefit of Skype consultation as it saved time and cost of travel, stating that 'I would love to do more of my appointments that way'. Patients using telemedicine for psychology appointments provided verbal feedback. All three patients felt very satisfied with the remote service since they could not have accessed this specialist care closer to home. Patients also commented that they would not have been able to make the regular journey to our centre in order to access the expertise of the dedicated IF psychological support.

There were no changes in PN scripts after telemedicine consultation. Two of the 25 patients had a catheter-related bloodstream infection following commencement of telemedicine, at a rate comparable to that of the overall HPN population managed at this centre during the study period $(0.082$ per 1000 catheter days for telemedicine cohort vs 0.074 per 1000 catheters days for all patients). No other complications were reported in those managed by telemedicine, and no emergency admission or outpatient appointment was required following remote video consultation. One emergency visit was potentially avoided; the patient was seen via telemedicine consultation outside normal clinic hours; advice was given; and the patient was then reviewed electively in the clinic.

\section{DISCUSSION}

In this prospective study, we demonstrate that telemedicine is both feasible and safe in managing HPNdependent patients cared for at a national IF centre. Notably, remote video consultation was easily implemented and had saved time and miles travelled for patients living far from the centre. Few studies have evaluated the impact of telemedicine in the HPN cohort and, to our knowledge, this is the first report concerning remote psychological management for this group of patients.

For a tertiary-care service like IF, telemedicine could be a solution to help address any geographical and/ or economical barriers. Most research studies in the literature have concluded that telemedicine systems are cost-effective ${ }^{11}$ and cost-saving in travel expense for patients. ${ }^{12}$ Other HPN centres outside of the UK have reported the use of telemedicine to help address large distances between the patients' homes and the IF centre, ${ }^{12-14}$ for example, Canadian colleagues reported using video-conferencing in 21 patients, of whom 
more than 50\% lived in remote or rural areas, with an associated estimated cost saving of $724 \$$ for each consultation. ${ }^{13}$ In addition to economic advantages, early remote evaluation via telemedicine can also prevent hospitalisations, emergency visits and catheter infections. ${ }^{12}$ In our experience, telemedicine was safe and much appreciated by patients, with a reduction in the need to travel to the hospital, so avoiding long journeys. It can also free up patients' valuable time, particularly for those requiring many nights of PN and having to fit hospital appointments between PN connections and disconnections. Moreover, telemedicine may remove the pressure on family members required to travel with patients for an appointment, although further work is required to gauge family members' satisfaction with the telemedicine service.

The various impacts of HPN, along with underlying disease, often lead to depression and/or anxiety. ${ }^{15-17}$ We offered remote video consultation within the IF psychology service to patients who live further away and/or who have less access to independent means of transportation. Similar specialised IF psychology services are rarely available locally to patients and if they have started an intervention while an inpatient, it is good practice to be able to complete this when they are discharged and with the same psychologist. ${ }^{18}$ With the telemedicine system, we have been able to offer a flexible psychological approach in terms of frequency of sessions according to the patient's needs with positive outcomes being achieved. Two patients were able to fully complete the psychological interventions required via Skype sessions up to discharge, and one patient is still using the telemedicine service and regularly reports that it is invaluable for well-being.

A weakness of our study is the low number of patients, making it impossible to draw solid conclusions. Although of great use for some, telemedicine is not suitable for every patient. Access and ability to use an electronic device are a prerequisite. ${ }^{19}$ Notably, clinician satisfaction of the telemedicine consultation was somewhat higher than that of patients; it is difficult to delineate the exact reason or reasons for this discrepancy. The clinical team's satisfaction was based on the ability to conduct a safe and reliable consultation, whereas patients were asked to rate their general satisfaction of the consultation, which may relate to the fact that patients may prefer the familiarity of face-toface appointments. Furthermore, the small number of patients using the telemedicine service for psychology consultations precluded meaningful conclusions from any patient satisfaction data. We therefore now aim to use a qualitative research approach to explore patients' and clinicians' attitudes to the telemedicine service in greater detail. In particular, further work is required to determine whether obviating the need to travel has a definite impact on quality of life when balanced against loss of face-to-face contact with the clinical team. ${ }^{20}$ Occasionally, in other studies, medical professionals have reported a lack of confidence in clinical judgement and in making a diagnosis via telemedicine. ${ }^{12} 1321$ However, we did not note any clinical limitations to using telemedicine in this cohort of patients and now aim to offer this initiative more widely to patients under our care.

\section{CONCLUSION}

HPN patient care remains highly specialised and is offered in relatively few locations. Inevitably, some patients will reside at a significant distance from the clinic. In selected HPN-dependent patients, telemedicine offers patient-centred and improved access to quality care. In the future, technological progress will help increase remote consultation services at our and possibly other centres, with the aim of reducing patients' need to travel.

Acknowledgements Thank you to all of the participants who took part in the study.

Contributors AB, SL and MIT contributed to conception/design of the research; JA, AA, MIT, AT, GL and KF contributed to the acquisition, analysis or interpretation of the data; AC, $\mathrm{AB}$ and JA drafted the manuscript; $\mathrm{AC}, \mathrm{AB}, \mathrm{JA}, \mathrm{MIT}$ and SL critically revised the manuscript; AC, MIT and SL agreed to be fully accountable for ensuring the integrity and accuracy of the overall content.

Funding This research was funded solely by the Salford Royal Foundation Trust Intestinal Failure Unit.

Competing interests None declared.

Patient consent for publication Not required.

Provenance and peer review Not commissioned; externally peer reviewed.

Data availability statement Data are available upon reasonable request.

\section{ORCID iDs}

Anabelle Cloutier http://orcid.org/0000-0003-0543-2729

Ashley Bond http://orcid.org/0000-0002-3237-4782

\section{REFERENCES}

1 Fleming CR, Remington M. Intestinal Failure. In: Hill GL, ed. Nutrition and the surgical patient. Edinburgh: Churchill Livingston, 1981: P.219-235.

2 Dibb M, Teubner A, Theis V, et al. Review article: the management of long-term parenteral nutrition. Aliment Pharmacol Ther 2013;37:587-603.

3 Pironi L, Arends J, Baxter J, et al. ESPEN endorsed recommendations. definition and classification of intestinal failure in adults. Clin Nutr 2015;34:171-80.

4 Pironi L, Arends J, Bozzetti F, et al. ESPEN guidelines on chronic intestinal failure in adults. Clinical Nutrition 2016;35:247-307.

5 British intestinal failure alliance (BIFA) position statement 2016 home parenteral nutrition (HPN).

6 Brandt CF, Hvistendahl M, Naimi RM, et al. Home parenteral nutrition in adult patients with chronic intestinal failure: the evolution over 4 decades in a tertiary referral center. JPEN $J$ Parenter Enteral Nutr 2017;41:1178-87.

7 Mundi MS, Pattinson A, McMahon MT, et al. Prevalence of home parenteral and enteral nutrition in the United States. Nutr Clin Pract 2017;32:799-805.

8 Smith T, Naghibi M. Artificial Nutrition Support in the UK 2005-2015. Adult Home Parenteral Nutrition \& Home Intravenous Fluids BANS Report; 2016. 
9 Le LB, Rahal HK, Viramontes MR, et al. Patient satisfaction and healthcare utilization using telemedicine in liver transplant recipients. Dig Dis Sci 2019;64:1150-7.

10 Langley GL, Moen R, Nolan KM, et al. The improvement guide: a practical approach to enhancing organizational performance. 2nd Edition. San Francisco, California, USA: Jossey-Bass Publishers, 2009.

11 de la Torre-Díez I, López-Coronado M, Vaca C, et al. Cost-Utility and cost-effectiveness studies of telemedicine, electronic, and mobile health systems in the literature: a systematic review. Telemed J E Health 2015;21:81-5.

12 Kim H, Spaulding R, Werkowitch M, et al. Costs of multidisciplinary parenteral nutrition care provided at a distance via mobile tablets. JPEN J Parenter Enteral Nutr 2014;38:50S-7.

13 Saqui O, Chang A, McGonigle S, et al. Telehealth videoconferencing: improving home parenteral nutrition patient care to rural areas of Ontario, Canada. JPEN J Parenter Enteral Nutr 2007;31:234-9.

14 Smith CE, Spaulding R, Piamjariyakul U, et al. mHealth clinic appointment PC tablet: implementation, challenges and solutions. J Mob Technol Med 2015;4:21-32.
15 Huisman-de Waal G, Schoonhoven L, Jansen J, et al. The impact of home parenteral nutrition on daily life-A review. Clinical Nutrition 2007;26:275-88.

16 Roskott AMC, Huisman-de Waal G, Wanten GJ, et al. \& Hoekstra-Weebers J.E.H. Screening for psychosocial distress in patients with long-term home parenteral nutrition. Clinical Nutrition 2013;32:396-403.

17 Winkler MF, Hagan E, Wetle T, et al. An exploration of quality of life and the experience of living with home parenteral nutrition. JPEN J Parenter Enteral Nutr 2010;34:395-407.

18 Ablett J, Vasant DH, Taylor M, et al. Poor social support and unemployment are associated with negative affect in home parenteral nutrition-dependent patients with chronic intestinal failure. JPEN J Parenter Enteral Nutr 2019;43:534-9.

19 Chaet D, Clearfield R, Sabin JE, et al. Ethical practice in telehealth and telemedicine. J Gen Intern Med 2017;32:113640.

20 Wolf JAet al. Defining patient experience. Patient Experience Journal 2014;1:7-19.

21 Chambers A, Hennessy E, Powell-Tuck J. Longitudinal trends in quality of life after starting home parenteral nutrition: a randomised controlled study of telemedicine. Clinical Nutrition 2006;25:505-14. 